\title{
Automatic Skin Color Beautification
}

\author{
Chih-Wei Chen, Da-Yuan Huang, and Chiou-Shann Fuh \\ Department of Computer Science and Information Engineering \\ National Taiwan University, Taipei, Taiwan \\ \{d95013,r97022, fuh\}@csie.ntu.edu.tw
}

\begin{abstract}
In this paper, we propose an automatic skin beautification framework based on color-temperature-insensitive skin-color detection. To polish selected skin region, we apply bilateral filter to smooth the facial flaw. Last, we use Poisson image cloning to integrate the beautified parts into the original input. Experimental results show that the proposed method can be applied in varied light source environment. In addition, this method can naturally beautify the portrait skin.
\end{abstract}

Keywords: Skin beautification, color-temperature-insensitive, bilateral filter, Poisson image cloning.

\section{Introduction}

Recently, digital still camera becomes more widespread. To provide more capability for users and satisfy their different requirements, many techniques have been integrated into digital still camera.

Skin beautification is the technique to smooth the skin of the photographed portrait. The result of skin beautification is preferable to the original one. Many consumer cameras have embedded the function of skin beautification. Besides, there are many approaches proposed to deal with skin beautification. However, most of these techniques rely on accurate skin color detection.

In this paper, we propose an original framework based on color-temperature- insensitive skin-color detection. Then, we use the characteristic of bilateral filter, which can preserve the significant image edge and can smooth the low gradient parts in given image, to polish the selected skin regions. Finally, to satisfy human perception, we apply Poisson seamless cloning algorithm to integrate the polished part into original image.

\section{Backgrounds}

\subsection{Skin Color Detection}

There are many skin color detection algorithms proposed to extract the skin parts from given image [1] [2]. Most of these approaches use statistical method to find out

F. Huang and R.-C. Wang (Eds.): ArtsIT 2009, LNICST 30, pp. 157-164, 2010.

(C) Institute for Computer Sciences, Social-Informatics and Telecommunications Engineering 2010 


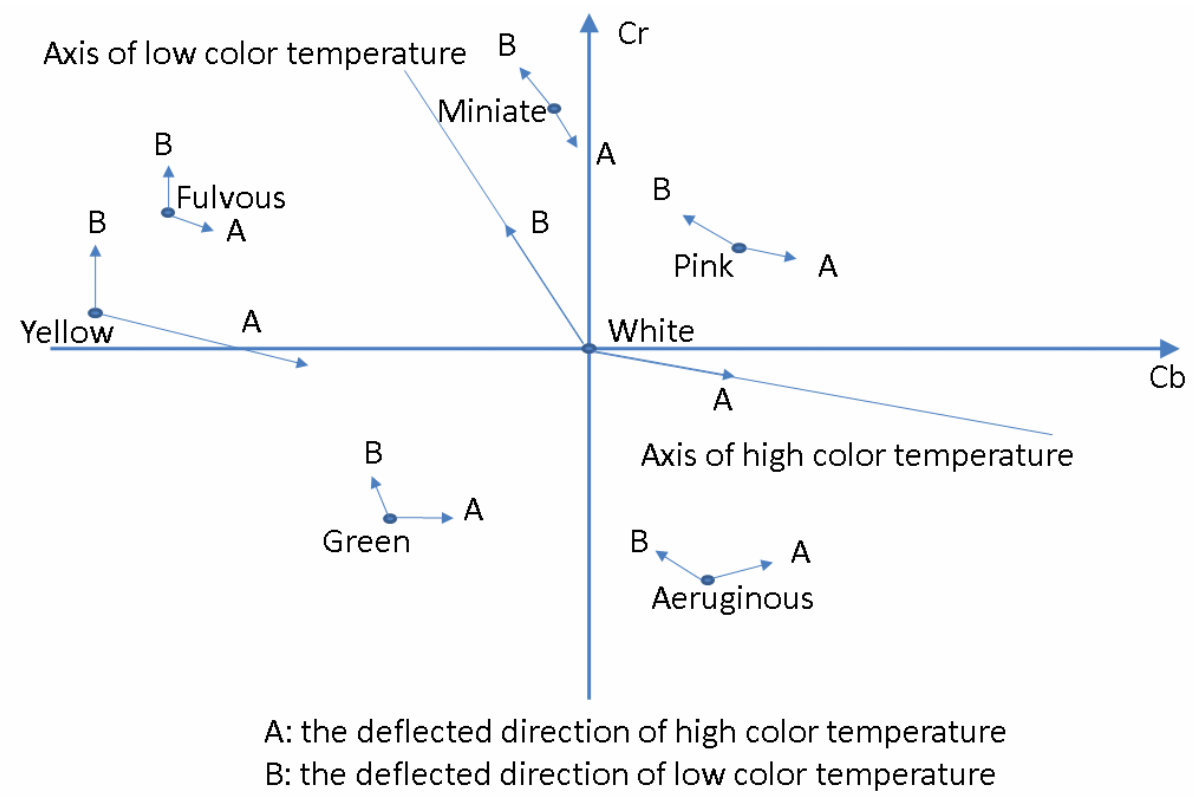

Fig. 1. The experimental data of color patches under different light sources [3]

the boundary of human skin color in specific color space. However, skin color is sensitive to color temperature. The relation between color and color temperature is shown in Fig. 1.

To lower the effect of color temperature, the better for us to use face detection algorithm as preprocessing step. Then, we can determine the characteristic of skin color from the abovementioned face detection.

\subsection{Face Detection}

To extract human face from given image, many algorithms have been proposed. Viola et al. proposed an efficient face detection approach [4]. There are three main principles in their algorithm, including the integral image, Ada boost, and cascade classifier. Furthermore, Lienhart et al. proposed Haar-like features for object detection [5]. In this paper, we apply Lienhart's frontal face classifier [6] and Hameed's eye classifier [7] to extract human face and human eyes from given image respectively. The objective of human eye detection is to subtract the effect of non-skin tone element from detected human face. The result of human face detection and human eye detection is shown in Fig. 2(b). The white color in Fig. 2(c) is the region of interest, which is a rough skin-color data. 


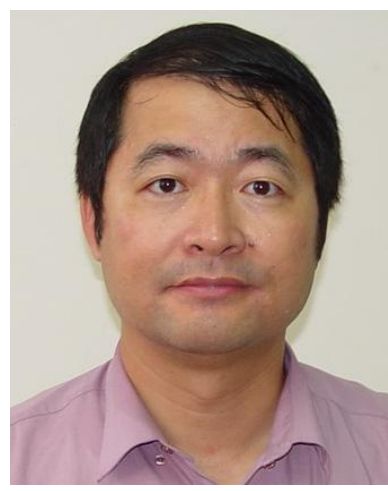

(a)

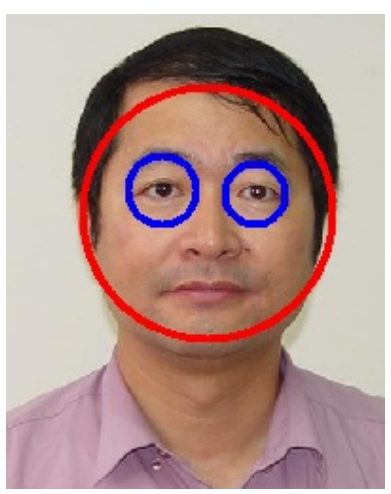

(b)

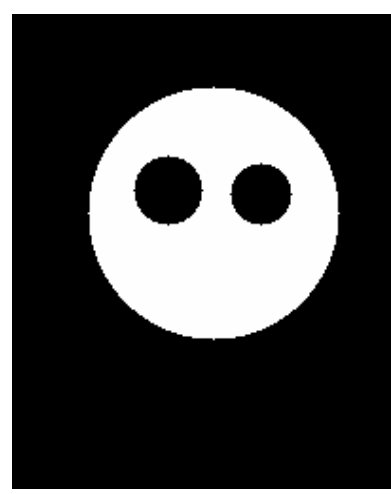

(c)

Fig. 2. (a) Input image. (b) Red circle and blue circle mean face region and eyes region respectively. (c) White region represents interested skin color.

\subsection{Bilateral Filter}

The bilateral filter is a nonlinear technique that can smooth the input image while preserving its main edges [8] [9]. With a Gaussian function $g_{\sigma}(x)=\exp \left(-x^{2} / \sigma^{2}\right)$ the bilateral filter of image $I$ at pixel $p$ is defined by:

$$
\begin{aligned}
B F(I)_{p} & =\frac{1}{w} \sum_{\mathrm{q} \in I} g_{\sigma_{s}}(\|p-q\|) g_{\sigma_{r}}\left(\left|I_{p}-I_{q}\right|\right) I_{q}, \\
w & =\sum_{q \in I} g_{\sigma_{s}}(\|p-q\|) g_{\sigma_{r}}\left(\left|I_{p}-I_{q}\right|\right),
\end{aligned}
$$

where $w$ is a normalized factor; $g_{\sigma_{s}}$ and $g_{\sigma_{r}}$ are typical Gaussian kernel functions; and $p$ and $q$ indicate spatial locations of pixels.

\subsection{Seamless Cloning}

The objective of Poisson seamless cloning is to find out a function, which makes the gradients of target image closest to the gradients of source image. We implement the discrete Poisson solver [10] to obtain finished result of skin beautification. In this paper, the guidance field vector $v$ is

$$
v=\nabla g
$$

where $\nabla g$ means the gradient of source image. 


\section{Our Proposed Method}

The proposed framework is shown in Fig. 3.

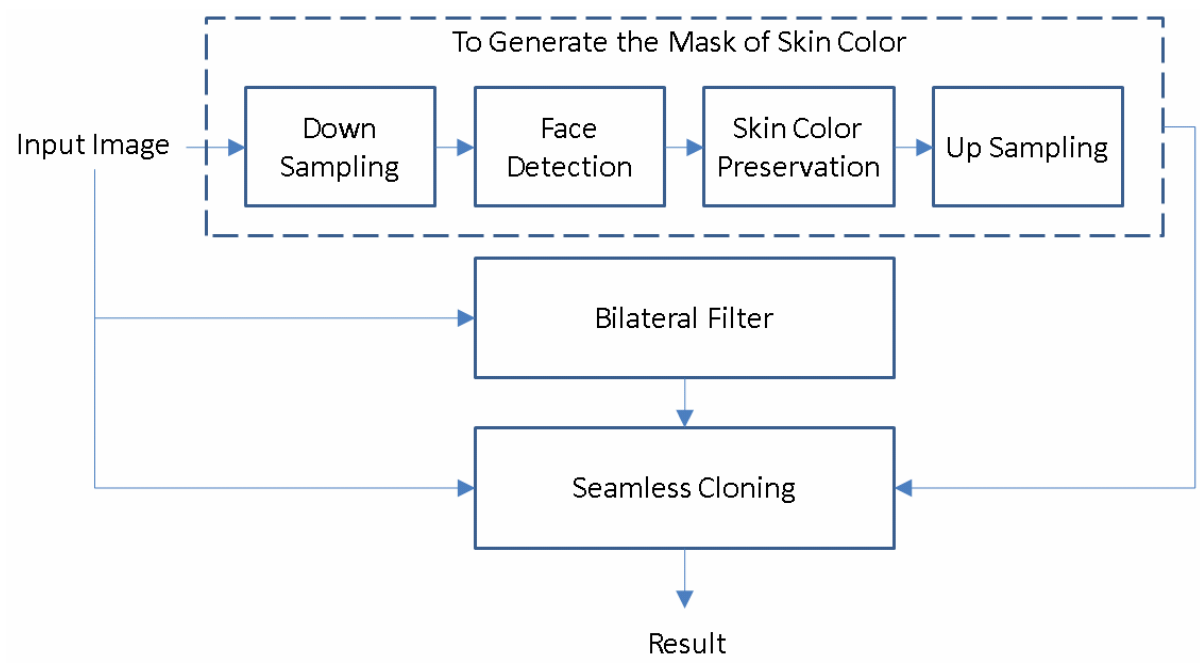

Fig. 3. System flowchart

We use down-sampling approach to lower the effect of facial flaw (e.g. scar, zit, spot, and so on). Besides, to downsize the input image can improve the efficiency of face detection. After face detection, the interested skin parts have been preserved, the sampling result is shown in Fig. 2(c). To achieve color-temperature-insensitive skincolor preservation, we translate the interested skin parts into $\mathrm{YCbCr}$ color space. Then, we calculate each mean value and standard deviation of $\mathrm{Y}, \mathrm{Cb}$, and $\mathrm{Cr}$ respectively. The abovementioned procedure is shown in Fig. 4. To prevent the effect of extreme value, we skip $10 \%$ of the head and tail in $\mathrm{Y}, \mathrm{Cb}$, and $\mathrm{Cr}$ histogram.

The skin-color mask of image $I$ at pixel $p$ is defined by

$$
M\left(I_{p}\right)=\left\{\begin{array}{cc}
1, & \text { if } \mu_{Y}-2 \sigma_{Y} \leq Y\left(I_{p}\right) \leq \mu_{Y}+2 \sigma_{Y} \\
& \text { and } \mu_{C b}-2 \sigma_{C b} \leq C b\left(I_{p}\right) \leq \mu_{C b}+2 \sigma_{C b} \\
& \text { and } \mu_{C r}-2 \sigma_{C r} \leq C r\left(I_{p}\right) \leq \mu_{C r}+2 \sigma_{C r} \\
0, & \text { otherwise, }
\end{array}\right.
$$

where $Y\left(I_{p}\right), C b\left(I_{p}\right)$, and $\operatorname{Cr}\left(I_{p}\right)$ mean the $\mathrm{YCbCr}$ value of image $I$ at pixel $p$ respectively; $\mu_{Y}, \mu_{C b}$, and $\mu_{C r}$ represent the mean value of interested region in $\mathrm{YCbCr}$ color space respectively; $\sigma_{\mathrm{Y}}, \sigma_{\mathrm{Cb}}$, and $\sigma_{\mathrm{Cr}}$ mean the standard deviation value of interested region in $\mathrm{YCbCr}$ color space respectively. 


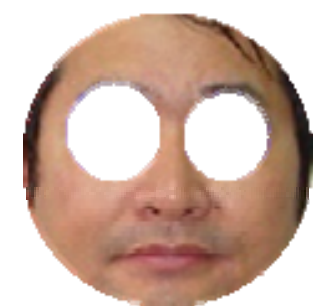

(a)

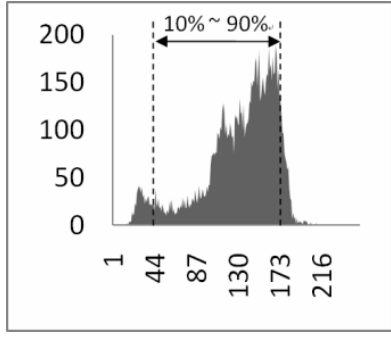

(b)

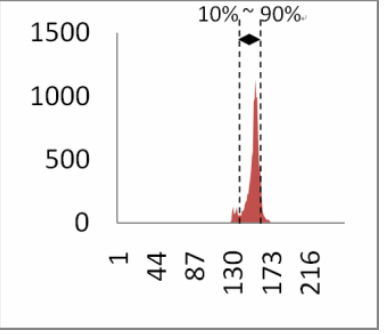

(c)

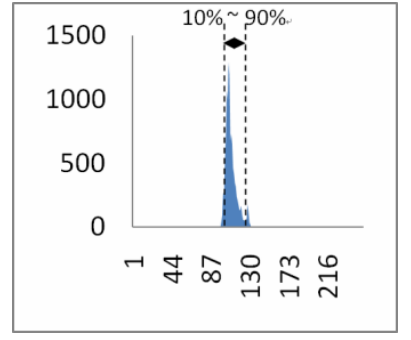

(d)

Fig. 4. (a) Region of interest. (b) The Y-channel of (a). (c) The Cr-channel of (a). (d) The Cbchannel of (a).

The abovementioned mask is generated in downsized form due to the downsampled input image. To accomplish the skin-color mask generation, we apply bilinear interpolation to adjust the size of the downsized form to equal to the size of input image.

Concurrently, we apply bilateral filter to smooth the original input image. The result of bilateral filter is called base image. The result of bilateral filter is similar to the output of low-pass filter. Moreover, bilateral filter can preserve the image edge simultaneously.

Finally, we integrate the input image, mask of skin-color, and the base image obtained from bilateral filter algorithm into result image. In this paper, we apply Poisson image cloning as image integration algorithm. The advantage of Poisson image cloning is to integrate the given image into harmonious image, even if there are few misdetections or false-alarms in the skin-color mask.

\section{Experimental Result}

To prevent the influence of color temperature, we use flexible method to determine the skin-color boundary. The performance of our skin-color detection is stable in various color temperatures. Table 1 shows the results of the proposed method. The size of input image is $349 \times 419$, and the $\sigma_{s}$ and the $\sigma_{r}$ in bilateral filter is set to 60 and 0.1 respectively. 
Table 1. The skin-color mask and beautification result of our proposed method in various color temperatures

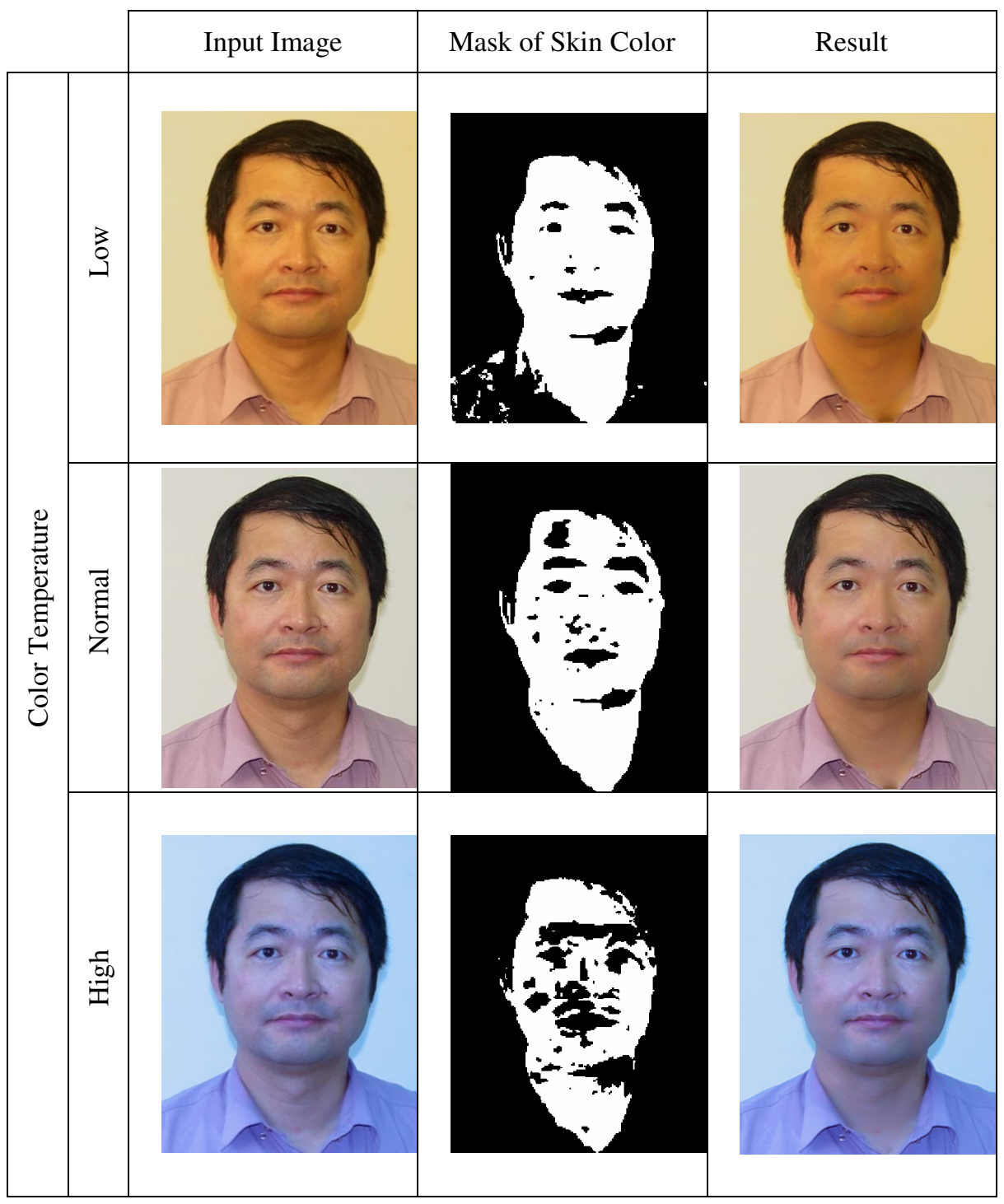

According to human perception, we can generate a touching portrait by smoothing the blemish (e.g. facial wrinkle, sweat pore, rash, and so on) of human face. Besides, our approach can be applied to lower the image noise in portrait photography. Table 2 shows the result of skin beautification in normal color temperature. 
Table 2. The results of skin beautification in normal color temperature

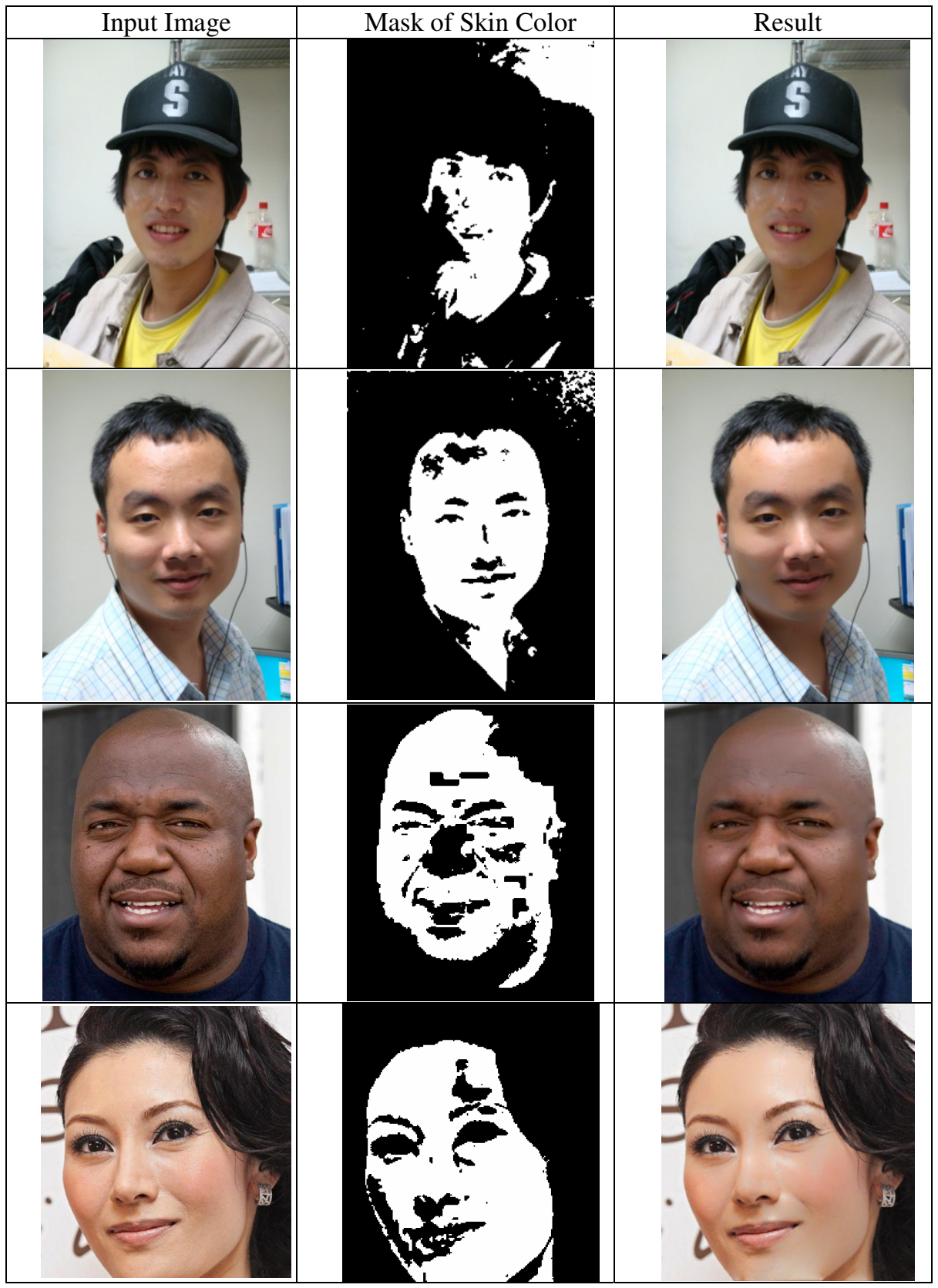




\section{Conclusion}

To judge the performance of skin beautification is quite subjective. In this paper, we propose an automatic approach, which is color-temperature-insensitive, to extract skin-color from given image. Then, we lower the blemish in interested part. There still are many challenging topics, including automatic skin tone adjustment in various light sources, automatic make up, automatic skin beautification in video stream, and so on.

Acknowledgements. This research was supported by the National Science Council of Taiwan, under Grants NSC 94-2213-E-002-032 and NSC 95-2221-E-002-276-MY3, by EeRise Corporation, EeVision Corporation, Primax Electronics, Altek, Alpha Imaging, Utechzone, TRI, Genesys Logic, Lite-on, and Syntek Semiconductor.

\section{Reference}

1. Tomaz, F., Candeias, T., Shahbazkia, H.: Fast and accurate skin segmentation in color images. In: Proceedings of First Canadian Conference on Computer and Robot Vision, 2004, pp. 180-187 (2004)

2. Kukharev, G., Nowosielski, A.: Visitor Identification - Elaborating Real Time Face Recognition System. Short Communication Papers Proceedings of WSCG 2004, pp. 157-164 (2004)

3. Yung-Cheng, L., Wen-Hsin, C., Ye-Quang, C.: Automatic white balance for digital still camera. IEEE Transactions on Consumer Electronics 41, 460-466 (1995)

4. Viola, P., Jones, M.J.: Robust Real-Time Face Detection. Int. J. Comput. Vision 57, 137154 (2004)

5. Lienhart, R., Maydt, J.: An extended set of Haar-like features for rapid object detection. In: Proceedings of 2002 International Conference on Image Processing, vol. 901, pp. I-900-I903 (2002)

6. Lienhart, R.: Stump-based 20x20 gentle adaboost frontal face detector, http: / /www. lienhart.de/

7. Hameed, S.: Stump-based 20x20 frontal eye detector, http://umich.edu/ shameem

8. Tomasi, C., Manduchi, R.: Bilateral filtering for gray and color images. In: Sixth International Conference on Computer Vision, 1998, pp. 839-846 (1998)

9. Paris, S., Kornprobst, P., Tumblin, J., Durand, F.: A gentle introduction to bilateral filtering and its applications. In: ACM SIGGRAPH 2008 classes. ACM, Los Angeles (2008)

10. P'erez, P., Gangnet, M., Blake, A.: Poisson image editing. ACM Trans. Graph. 22, 313$318(2003)$ 